\title{
Grenzen und Technologie
}

\author{
Holger Pötzsch
}

\begin{abstract}
Dieser Beitrag behandelt Staatsgrenzen und Technologie. Nach einem Überblick über den Forschungsstand wendet sich der Text möglichen Implikationen einer verstärkten Nutzung von Biometrie, Netzwerküberwachung, algorithmischer Prädiktion und Automatisierung in heutiger Grenzsicherung zu. Es werden Potenziale für Überwachung und Kontrolle aufgezeigt und sodann kritisch von der Perspektive verorteter Praktiken her beleuchtet. Zuletzt wird für einen Identität konstituierenden Charakter heutiger hochtechnisierter Grenzregime argumentiert, die nicht nur scheinbar gegebene Subjekte prozessieren, sondern auch durch prädiktive Analytik aktiv an deren Erschaffung teilhaben.
\end{abstract}

\section{Schlagwörter}

Biometrie, Netzwerke, Algorithmen, Prädiktion, Überwachung

\section{Grenzen und Staatsgrenzen: eine Einleitung}

Grenzen sind überall. Ohne Grenzen gäbe es keine Ordnung, keine Bedeutung, kein ich und kein du. Martin Heintel et al. (2018, S. 1) legen auf dieser Grundlage folgende Definition vor: „Eine Grenze ist eine gedachte oder abstrakte Linie, anhand welcher Unterscheidungen getroffen und Dinge durch Differenz identifiziert werden“. Um die Welt überhaupt wahrnehmbar und mitteilbar zu machen, scheinen Grenzen unabdingbar. Wahrnehmung, Bedeutung, Kommunikation, Identität sind in dieser Sicht alle von expliziten oder impliziten Grenzziehungen und -setzungen abhängig. In diesem weitesten Sinne sind Grenzen „selektierende und kategorisierende Hierarchisierungsinstitutionen“ (Heimeshoff et al. 2014, S. 17), die die Grundlage allen menschlichen Seins und Wirkens bilden. Nach Chris Perkins und Chris Rumford (2013, S. 272) sind Grenzen daher nicht nur als Begrenzungen und Hindernisse, sondern auch als Ressourcen zu verstehen - als „practical everyday methods for navigating indeterminate pluralities“.

Dieses Kapitel widmet sich einer Sonderform der so definierten Grenze - der politischen Staatsgrenze. Zunächst wird dem ,klassischen' geopolitischen Grenzbegriff nachgegangen, gefolgt von einer Argumentation für dessen - trotz Globalisierung und Überstaatlichkeit - andauernde Relevanz für ein Verständnis aktueller politischer und gesellschaftlicher Dynamiken. Die Staatsgrenze erscheint hier zum einen als Filter zur Kontrolle von Strömen von Menschen, Gütern, Rohstoffen und Ähnlichem, zum anderen als eine Orientierungs- und Kontaktzone, die Unterscheidungen und Austausch möglich macht. Im Anschluss daran werden Argumente für einen technologisch fundierten enträumlichten Grenzbegriff vorgebracht. Dieser versteht die Grenze als in die Territorien von Staaten gefaltet ${ }^{1}$ und in steigendem Maße an individuelle Körper geheftet und oft von nichtstaatlichen Akteuren realisiert. Diese enträumlichte, verkörperte und privatisierte Staatsgrenze ist das Resultat von biometrischen und digitalen

1 Der Begriff geht auf Gilles Deleuze (1992) zurück, der die Falte als eine dritte geometrische Kategorie zwischen den Alternativen Linie und Fläche denkt. Für eine Anwendung in Grenzstudien vgl. z.B. Nicholas Henry (2018, insb. S. 22-23). 
Überwachungs- und Profilierungssystemen, die als weitgehend unsichtbare Hintergrundprozesse nahezu ununterbrochen und überall ablaufen. Die Grenze verliert damit ihre territoriale Gebundenheit und verlagert sich vom Feld staatlicher Souveränität zu dem einer globalen Sicherheitskonzeption. Sie wird dynamisch und individualisiert und ändert ihre Durchlässigkeit und Wirkungsweise abhängig vom Grad der Konformität der jeweils prozessierten Subjektivität. Diesem Verhältnis zwischen Grenzfunktionen, Technologie und menschlichen Subjekten gilt das Hauptaugenmerk dieses Beitrages.

Nach einer Beschreibung der soziotechnischen Affordanzen ${ }^{2}$ heutiger hochtechnologischer Grenzen wird dann ein kritischer Blick auf die situierten Praktiken konkreter Grenzregime gerichtet, durch welche Potenziale für Überwachung und Kontrolle verwirklicht, aber eben immer auch unterlaufen werden. Hier gilt es der Kontingenz technischer Lösungen nachzugehen und mögliche Mängel, Fehler, Missbrauch sowie zeitliche und kulturelle Gebundenheiten auszuloten, die selbst scheinbar perfekte Modelle und Pläne als abhängig von konkreten Praktiken der Implementierung entlarven. Wie bereits früher gezeigt (Pötzsch 2015, S. 112), relativiert diese Bottom-up-Perspektive staatliche „ambitions of comprehensive surveillance, management, and control“ und richtet Aufmerksamkeit auf die „messy realities of [...] incremental day-to-day implementation “ und ermöglicht es daher, dem, was Heimeshoff et al. (2014, S. 20) eine "Schere zwischen Programmatik und Praxis“ nennen, produktiv nachzugehen. Zuletzt wird dann für eine konstituierende Wirkung heutiger Grenzen, die nicht nur gegebene Subjektivität prozessieren, sondern auch an deren Hervorbringung aktiv teilhaben, argumentiert.

\section{Staatsgrenzen und Technologie}

Einiges deutete in den 1990er-Jahren auf einen allmählichen Abbau staatszentrierter Vorstellungen und Praktiken hin. Mit dem Ende des Ost-West-Konfliktes, zunehmendem Freihandel und einer stetigen Erweiterung der Europäischen Union und vergleichbarer überstaatlicher Organe begannen Grenzforscher einen jahrzehntealten Konsensus herauszufordern. Bedeutete Globalisierung ein Ende staatlicher Macht im Sinne einer Weltordnung ausgehend vom Westfälischen Frieden? Ist die Geopolitik mit ihrem Fokus auf die Interaktion souveräner Containerstaaten in internationalen Relationen in einer territorial trap (vgl. Agnew 1994) gefangen, die sie außer Stande setzt, zentrale Entwicklungen sinnvoll zu deuten? Verlieren geopolitisch ausgerichtete Grenzstudien daher mehr und mehr ihre Berechtigung?

Tatsächlich werden heute Identitäten und Zugehörigkeiten häufiger als früher transnational und durch globale Medien vermittelt ausgehandelt (vgl. Appadurai 1996; Dahinden 2009). Auch haben Manuel Castells (1996) mit seiner Beschreibung eines Überganges zu einer Netzwerkgesellschaft und Michael Hardt und Antonio Negri (2000) mit ihrer Theorie eines grenzenlosen Imperiums, das keine traditionelle Außenpolitik mehr kennt, wichtige Dynamiken erfasst, die auch die Funktionsweisen und Effekte spätmoderner Grenzregime umfassen. Gleichzeitig wird aber auch durch erneutes Errichten von Grenzmauern und -zäunen oder anhaltende Flüchtlingskrisen deutlich, dass traditionelle Staatsgrenzen und staatszentrisches

2 Der von James J. Gibson (1979) eingeführte und von Donald Norman (1988) popularisierte Begriff affordance wird häufig mit Angebotscharakter oder Möglichkeitshorizont übersetzt. Er beschreibt die im Design von Objekten angelegten Gebrauchsmöglichkeiten, die systematisch bestimmte Handlungen ermöglichen und andere erschweren. Daher macht der Begriff eine Problematisierung menschlichen Agens mit Blick auf agentische Potenziale von Tieren sowie technischer und anderer Objekte möglich (vgl. Pötzsch 2017, insb. S. 164). 
Denken und Handeln auch weiterhin von großer Bedeutung sind. Auch heute greifen staatliche Grenzen auf entscheidende Weise in die Lebenswelten und Entfaltungsmöglichkeiten vieler Menschen ein.

Liam O'Dowd (2010) warnt daher vor einem vorschnellen Konsens in Grenzstudien. Er spricht vor dem Hintergrund einer anhaltenden globalen Akzeptanz von Staatsgrenzen als „most widely recognised and institutionalised dividers of world space“ (ebd., S. 1031), dass das Fach oft unkritisch von einem historisch nicht belegbaren, sukzessiven Verfall von Staatsgrenzen ausgehe, die, wie gerne angenommen wird, ihren Zenit überschritten hätten. „This reluctance [of border studies to adopt a proper historical outlook] encourages a form of pseudohistory or ,epochal thinking' which disfigures perspectives on the present", so O'Dowd (ebd., S. 1032). Stattdessen müsse es darum gehen, den sich ständig ändernden Wirkungsweisen und Ausformungen staatlicher Macht nachzuspüren, sie historisch zu kontextualisieren und kritisch zu hinterfragen. Der Einfluss traditioneller souveräner Containerstaaten mit klar definierten territorialen Grenzen verschwinde in Folge nicht, er nehme lediglich neue Formen an.

Technologische Neuerungen spielen für die Änderung von nationalstaatlichen Grenzregimen eine große Rolle. In seiner grundlegenden Studie zur Rolle klassischer Massenmedien für die Konstruktion homogener nationaler Identitäten innerhalb klar abgegrenzter staatlicher Einheiten hat Benedict Anderson (1991) einen solchen Zusammenhang beschrieben. Während eine mittelalterliche Ordnung von multiplen Zentren dominiert war, die mit zunehmendem Abstand schnell an Bindungskraft verloren, in Randzonen ausfaserte und dort oft von überlappenden und geteilten Loyalitäten gekennzeichnet war, definieren sich moderne Nationalstaaten über klare Außengrenzen, die einen homogenen Innenraum eindeutig von umgebenden Territorien abgrenzen. Anderson beschreibt die Rolle moderner Massenkommunikationsmittel wie Zeitungen und Romane für die Etablierung einer solchen inneren Homogenität, während Arjun Appadurai (1996) darlegt, wie diese innere Stabilität durch elektronische Medien und Massenmigration zunehmend herausgefordert wird.

Tatsächlich kann es auf den ersten Blick den Anschein haben, als würden globale Medienkanäle und digitale Netzwerke ein traditionelles, territorial definiertes Staats- und Identitätsverständnis herausfordern (vgl. Appadurai 1996; Castells 1996). Leicht wie nie zuvor lassen sich heute territorial bedingte Grenzen medial überqueren. Identität und Zugehörigkeit wird zunehmend auf Grundlage global zugänglicher Kulturprodukte und Medientechnologien verhandelt und Staaten scheinen Einfluss auf diese Prozesse zu verlieren. Das beeinflusst auch, wie Grenzen heute imaginiert werden. Maria Rovisco (2010, S. 1028) zufolge erscheint ein „new border imaginary [...] underpinned by a cosmopolitan grammar of difference“ als eine brauchbare Alternative, um ein besseres, historisch fundiertes Verständnis europäischer Grenzen herausarbeiten zu können.

Heutigen, scheinbar Identitäten und Zugehörigkeiten auflösenden globalen Netzwerken liegt jedoch eine materielle Infrastruktur zugrunde, die über die Hintertür doch wieder, und in einigen Fällen sogar in verstärkter Form, staatlichen Einfluss und Kontrolle möglich macht (vgl. Starosielski 2015; Amoore 2018). Louise Amoore (2018) führt z.B. den Begriff cloud geography ein, um die Aufmerksamkeit auf diesen technisch-institutionellen Unterbau scheinbar virtueller globaler Netzwerke und Datenwolken zu lenken. Für sie ist es genau diese Infrastruktur, die klassische staatliche Macht und Herrschaft heute in wachsendem Ausmaß möglich macht: „[u]nderstood as a spatial arrangement, materialized in and through data 
centres, the abstract deterritorialized cloud is thus reterritorialized as an intelligible and governable entity" (ebd., S. 8).

Aus ähnlicher Perspektive haben Baumann et al. (2014) die Idee einer schwindenden souveränen Staatsmacht im Internet herausgefordert. Sie zeigen auf, wie Staaten als Reaktion auf die von Edward Snowden aufgedeckte NSA-Überwachungsaffäre verstärkt darauf bedacht sind, auch digital ihre Grenzen zu sichern und zu verstärken. Dies gilt nicht nur für offenbar autoritäre Systeme wie Iran oder China, sondern im allerhöchsten Grad auch für Einheiten wie die Europäische Union, die ebenfalls Versuche unternimmt, eigene Daten vor dem Zugriff amerikanischer und anderer Interessen zu schützen.

Solche Aktivitäten staatlicher und überstaatlicher Organe sind nicht ohne historische Präzedenz. Wie Daniel Joseph (2018) hervorhebt, plante Kanada in den 1970er-Jahren ein eigenes nationales Computernetzwerk - Trans-Canada Computer Communications Network -, um eine gangbare Alternative zu einem von amerikanischen Interessen dominierten und mit Geldern militärischer Forschung entwickelten ARPANET, dem Vorläufer des heutigen Internets, aufzubauen. Ähnliches gilt für das russische Runet, dessen noch zu Zeiten der UdSSR entwickelte Infrastruktur die Grundlage für die heutige russische Unabhängigkeit im digitalen Raum darstellt (vgl. Limonier 2017).

Trotz solcher fortdauernden Bedeutung staatlicher Akteure für grenzbezogene Prozesse und Praktiken im globalen und virtuellen Raum besteht heute weitgehende fachliche Einigkeit darüber, dass Grenzen per se multiperspektivische Gebilde und Prozesse darstellen und dass diese weit über staatliche Institutionen und physische Infrastruktur hinausreichen. Sie können daher nur fächerübergreifend untersucht werden und verlangen nach einer Kombination unterschiedlicher Methoden und Herangehensweisen, die verschiedene Disziplinen zusammenführen (vgl. Rumford 2012; Brambilla 2014; Nissel 2018; Pötzsch 2018). Wie Franck Billé (2018, S. 61) es ausdrückt, wird die Suche nach einer das Fach einenden „unifying grand theory“ daher heute weitgehend als illusorisch angesehen.

Tatsächlich haben neue Technologien wie digitale Netzwerke und Datenbanken staatliche Aufgaben und Macht nicht notwendigerweise reduziert, sondern verändert (vgl. Popescu 2011; Longo 2017). Diese scheinen heute nicht länger vornehmlich auf eine Homogenisierung nationaler Identitäten von oben herab und auf eine souveräne Kontrolle staatlicher Außengrenzen ausgerichtet zu sein, sondern streben zunehmend auch nach einer Regulierung und Kontrolle von abstrakten Identitätskategorien und Verhaltensmustern auf globaler Ebene (vgl. Amoore 2013; Pötzsch 2015). Zu diesem Zweck sind der Zugang zu und die Analyse von globalen Datenströmen und Datenbanken von größter Wichtigkeit. Alexander Galloway und Eugene Thacker (2007, S. 46) führen die Begriffe networked sovereignty, network sovereignty und distributed sovereignty ein, um solche Änderungen begrifflich zu erfassen. Während der erste Ausdruck technisch induzierte Änderungen etablierter staatlicher Praktiken beschreibt, umfasst der zweite ein völlig neues Verständnis von Souveränität, in dem Algorithmen, digitalen Netzwerken und Datenbanken eine eigene Form von Agens und Entscheidungsmacht zugeschrieben wird. Die Autoren beschreiben diese neue Form von Souveränität als „distributed“ (ebd., S. 46) - als unter zahlreichen dicht vernetzten Knotenpunkten verteilt und erst durch deren Zusammenwirken effektiv realisierbar.

In diesem Sinne ähnelt der Begriff der „distributed sovereignty“ von Galloway und Thacker (2007, S. 46) dem Souveränitätsverständnis Judith Butlers (2004). Sie verfolgt das Ziel, einen 
rein theoretischen Souveränitätsbegriff als von einer zentralen Staatsmacht über im Prinzip machtlose Untertanen ausgeübtes Herrschaftsverfahren, wie es beispielsweise in den Arbeiten Carl Schmitts (1922/2005) oder Giorgio Agambens (1998) hervortritt, auszuhebeln und durch ein praxisbezogenes alternatives Verständnis zu ersetzen. In Folge kann laut Butler (2004) Souveränität ohne aktive Mitwirkung der Beherrschten nicht existieren. Staatliche Macht benötigt staatlich sanktioniertes individuelles Handeln, um realisiert zu werden. Konsequenterweise richtet Butler ihre Aufmerksamkeit auf das tagtägliche Wirken von so genannten „petty sovereigns“ (ebd., S. 56), einzelnen Individuen, die mit staatlicher Macht versehen für diesen Staat ihre Mituntertanen regieren.

Butler beleuchtet durch diesen Perspektivenwechsel eine Dunkelstelle im Denken von sowohl Schmitt als auch Agamben - das Agens von Einzelpersonen in Prozessen souveräner Machtausübung. Wie Didier Bigo (2007) es unter Hinweis auf Michel Foucault (1977) und mit Blick auf heutige Grenzregime ausdrückt, hat Souveränität eine eigene microphysics of power, die auf dem Niveau individuellen Agierens lokalisiert ist und immer auch möglichen Widerstand miteinschließt: eine „resistance of the weak and their capacities to continue to be humane and to subvert the illusory dream of total control“ (Bigo 2007, S. 12). Souveränität fehlt daher, so Mark B. Salter (2011, S. 66), eine eigene Essenz. Sie ist davon abhängig, in „,stylized repetition of acts' of sovereignty" stetig neu artikuliert zu werden.

In ihrer Feldstudie in britischen Auffanglagern für Flüchtlinge zeigt Alexandra Hall (2012), wie Technologie auf die Handlungsweisen solcher petty sovereigns und der von ihnen verwalteten Menschen einwirkt. Ihre anthropologische Studie macht u.a. deutlich, dass staatliche Souveränität heute nicht nur durch Alltagspraktiken menschlicher, sondern auch nichtmenschlicher Akteure realisiert wird. Als solches erscheint es notwendig, Butlers petty sovereigns maschinelle Entsprechungen zur Seite zu stellen. Dies führt zu einer Komplizierung und Aufsplitterung des Agensbegriffs in komplexen soziotechnischen Netzwerken (vgl. Coole 2013; Hogan 2015; Pötzsch 2017, S. 12-17) und macht deutlich, dass unter der Bedingung einer distributed sovereignty in heutigen Grenzregimen Menschen und Maschinen Hand in Hand agieren und dadurch wechselseitig ihre jeweiligen Handlungsräume und -alternativen bedingen (siehe dazu auch Lindemann in diesem Band).

Vor diesem Hintergrund wird dieser Beitrag im Folgenden der Rolle neuer Technologien in heutigen global ausgerichteten und zunehmend autonomen Sicherheits- und Grenzregimen nachgehen. Hauptaugenmerk gilt den Implikationen biometrischer Verfahren sowie den möglichen Folgen neuer Überwachungs-, Profilierungs- und Sortierungstechnologien. Dann werden die identifizierten Systeme und ihre spezifischen Affordanzen vor dem Hintergrund von Alltagspraktiken kritisch perspektiviert und schließlich vor möglichen negativen Konsequenzen solcher Identitäten und Verhalten nicht nur beschreibenden, sondern diese auch aktiv konstituierenden digitalen Grenztechnologien gewarnt wird.

\section{Die enträumlichte Grenze: Körper, Algorithmen, Netzwerke}

Globaler Raum kann nicht nur als in Nationalstaaten aufgeteilt verstanden werden, sondern tritt ebenfalls als eine Reihe flexibler, sich überlappender territorialer und anderer Entitäten hervor, die sich u.a. durch gemeinsame Standards oder Regulierungssysteme definieren lassen (Walters 2011, Brambilla 2014, Pötzsch 2015). William Walters (2011) führt den Begriff der technischen Zone ein, um die Rolle von Technologie in diesen Prozessen hervorzuheben. Er 
argumentiert, dass für ein effektives Funktionieren von biometrischen Pässen, elektronischen Fingerabdruckregistern, oder international kompatiblen Datenbanken überstaatliche Standards und Institutionen notwendig sind. Dies führt ihn dazu, einer staatlich definierten globalen Arena ein im Prinzip grenzenloses Sicherheitsregime entgegenzustellen, in dem technische und andere Zonen neben traditionellen Nationalstaaten vermehrt zur Geltung kommen.

In einer ähnlichen Neueinteilung führt Benjamin H. Bratton (2015) den Begriff des stack ein, um solche Entwicklungen begrifflich fassbar zu machen. Er schlägt eine Aufteilung globalen Raumes in sechs miteinander verknüpfte Niveaus vor, die individuelle Nutzer und deren Praktiken ebenso einschließen wie Städte, virtuelle Datenwolken und die Erde selbst. Brattons Konzeption hat den Vorteil, dass sie scheinbar virtuelle Technologien mit konkreten geophysischen, biologischen und sozialen Prozessen in Verbindung bringt und somit einem Immaterialitätsmythos digitaler Netzwerke entgegentritt. Der vorliegende Beitrag rekurriert dennoch auf die Konzeption von Walters (2011), da diese eine klarere Perspektive auf Verknüpfungen zwischen globalen Strukturen und lokalen Praktiken mit spezifischem Fokus auf Grenztechnologien ermöglicht.

Walters' (2011, S. 55) „technological zones“ sind für ihre Realisierung wie auch für ihre mögliche Subversion von „technological work“ abhängig - den vielen, scheinbar unbedeutenden tagtäglichen Handlungen, die Technologie und soziotechnische Systeme instandhalten und überhaupt erst zum Funktionieren bringen. Seine Studie belegt, dass diese entgrenzten technologischen Zonen und die sie bedingende technologische Arbeit auch für die Regulierung und Steuerung globaler Güter-, Menschen- und Datenströme von entscheidender Bedeutung sind.

Zentrale technische Komponenten heutiger spätmoderner Grenz- und Sicherheitsregime sind biometrische Identifikationsverfahren, Überwachung und Speicherung digitaler Datenströme, vernetzte und kompatible Datenbanken, algorithmisch getriebene prädiktive Analytik und eine Tendenz zur Automatisierung und Privatisierung wichtiger staatlicher Funktionen (vgl. Amoore 2013; Pötzsch 2015; Longo 2017; auch Schwell in diesem Band). Diese technologisch bedingten Dynamiken weiten auf der einen Seite staatlichen Zugriff auf eine globale Arena aus und falten diesen auf der anderen Seite ins Innere etablierter Nationalstaaten hinein. Wie im Folgenden aufgezeigt wird, führt dieses Überschwappen von Ausnahmezonen sowohl in die Privatsphäre als auch in die Gänze staatlichen Raumes nicht nur zu einer Deterritorialisierung und Globalisierung, sondern auch zu einer Individualisierung und Verkörperung heutiger Grenzen.

\subsection{Biometrie und digitale Überwachung: die verkörperte Überallgrenze}

Die Anwendung biometrischer Verfahren bei Grenzkontrollen ist kein neues Phänomen. Im Gegenteil, ein ,Sortieren“ von Menschen auf Grundlage gewisser scheinbar einzigartiger körperlicher Eigenschaften war schon immer Teil von Grenzregimen. Was sich jedoch geändert hat, ist der Grad der Technisierung solcher Verfahren und daher deren Reichweite, Vernetzung und angenommene Genauigkeit (vgl. Maguire 2009). Wie u.a. Brigitta Kuster und Vassilis S. Tsianos (2013 S. 24; Fußnote 5) hervorheben, geht es biometrischer Grenztechnologie zum einen um eine Verifikation individueller Identitäten. Um herauszufinden, ob die grenzüberschreitende Person tatsächlich diejenige ist, für die sie sich ausgibt, werden über Identitätsdokumente zugängliche Daten mit körperlichen Merkmalen verglichen. Zum anderen geht es 
aber auch darum, herauszufinden, wer eine bestimmte Person ,wirklich' ist. Zu diesem Zweck müssen körpereigene biometrische Daten mit anderen zugänglichen Informationen und Datenbanken abgeglichen werden.

Biometrische Daten werden heute auf mit RFID-Technologie ${ }^{3}$ ausgerüsteten Ausweisen und Pässen festgehalten. An den smarten Grenzen staatlicher Einheiten wie der Europäischen Union oder den USA werden diese Daten dann an sogenannten E-Gates maschinell lesbar und können rasch mit körperlichen Merkmalen wie Gesichtsform, Iris oder Fingerabdrücken sowie zusätzlichen Information aus anderen Datenbanken verglichen werden. Dies ermöglicht es implizit als normativ angesehene Identitätskategorien schnell zu prozessieren und so die Effektivität etablierter Grenzregime durch Zugriff auf digitale Profile zu verbessern. In den Worten Benjamin B. Muellers (2008, S. 128): „[t]he biometric border constitutes an interface between the corporeal, or materially manifested self, the body, and the data-double, or dossier it represents“. Körper und in vernetzten Datenbanken gelagerte Identitätsmuster werden zusammengeführt und erleichtern so die Kontrolle und Steuerung von Menschenströmen.

Eine Kombination biometrischer Verfahren mit eng vernetzten Datenbanken wie NEXUS, Eurodac oder Schengen Information System II $^{4}$ machen auch zunehmend automatisierte Praktiken einer deterritorialisierten Grenzkontrolle wie pre-screening und upstream profiling möglich. Diese Methoden machen individuelle Körper zu Trägern der Grenze (vgl. Amoore 2006; Pötzsch 2015) und weiten Kontrollmöglichkeiten ins Innere von Nationalstaaten aus. Die an individuelle Körper und digitale Geräte geheftete biometrische und elektronisch vernetzte Grenze füllt die Gesamtheit staatlicher Räume aus und folgt Subjekten wohin auch immer sie sich bewegen. Wie Joseph Pugliese (2010, S. 26) es ausdrückt, hat sich damit der „capillary reach of state violence“ bis in die "quotidian sites of civilian life“ ausgeweitet. Das Resultat ist ihm zufolge ein allgegenwärtiger Zustand potenzieller Verfolgung, der eine endgültige Überquerung der Grenze und damit eine Ankunft im Inneren unmöglich macht. Btihaj Ajana (2013, S. 6) zufolge führt dies zu einem „spillover from exceptional spaces [...] to the general body of humanity “, der die Ausnahme normalisiert und sie in eine implizit normative, allumfassende Praxis verwandelt.

Die verstärkte Anwendung biometrischer Verfahren und deren Kombination mit vernetzten Datenbanken dehnt also die vormals territorial definierte Grenze aus und macht sie, durch ihre Verkörperung, im Prinzip allgegenwärtig und unüberquerbar. Gleichzeitig ist diese Grenze jedoch nicht für jedermann gleichermaßen fühl- und sichtbar. Wie Pugliese (2010), Ajana (2013) und Matthew Longo (2017) konstatieren, sind heutige Grenzen und ihre vielfältigen technisch induzierten Aktivitäten des Registrierens, Analysierens und Sortierens für hegemoniale Subjektivitäten weitgehend unsichtbar und folgenlos, während Individuen, die den jeweils gesetzten, kontingenten Normen nicht oder nur teilweise entsprechen, diese Grenzen wie mobile Gefäng-

3 RFID steht für radio-frequency identification device. RFIDs sind mit Sendern variabler Reichweite ausgestattete Mikrochips, die es möglich machen, Waren und Güter auf Abstand automatisch zu verfolgen und bei Bedarf umzuleiten. Ursprünglich im Container- und Postbetrieb genutzt, hat sich diese Technologie auf eine Vielzahl von Lebensbereichen ausgedehnt und wird heute in Kartenschlüsseln, Kopierkarten, elektronischen Fahrkarten und eben auch in biometrischen Pässen genutzt.

4 NEXUS ist eine kanadische-US-amerikanische Grenzkontrollinitiative, die es ausgewählten trusted travellers nach Aufnahme in eine Datenbank ermöglicht, die Grenze durch spezielle Kontrollposten rasch zu passieren. Eurodac ist ein elektronisches Fingerabdruckregister für Asylbewerber, das schnellen Datenabgleich zwischen nationalen Behörden von EU-Mitgliedsstaaten ermöglicht. Schengen Information System II ist eine gemeinsame Datenbank der Unterzeichnerstaaten des Schengener Abkommens, die für polizeiliche Ermittlungen und Einwanderungskontrolle genutzt wird. 
nisse mit sich herumtragen. Der Körper selbst wird so zum „carrier of the border“ (Amoore 2006, S. 347f.), zum stetigen soziopolitischen Stigma und wandelnden Beweis potenzieller Nichtzugehörigkeit (vgl. dazu auch Bruns in diesem Band).

\subsection{Grenzpraktiken: soziotechnische Potenziale und ihre Umsetzung}

Wie eben beschrieben, sind Biometrie, umfassende Überwachung digitaler Netzwerke, effektiv verknüpfte Datenbanken und algorithmisch getriebene Analytik heute Kernelemente von auf Effektivität getrimmten hochtechnisierten Grenzregimen. Das US-amerikanische NEXUSProgramm, britische e-Border-Initiativen sowie die Pläne der Europäischen Kommission für umfassende EU-Smart Border-Lösungen sind unterschiedliche Versuche, das Potenzial neuer technischer Systeme gewinnbringend auszunutzen. Die Funktionstüchtigkeit und Effizienz all dieser technischen Rahmen ist jedoch von entsprechenden Alltagspraktiken unterschiedlich situierter Individuen - Butlers petty sovereigns - abhängig.

Heimeshoff et al. (2014) rekurrieren auf das Konzept des borderwork von Rumford (2008), um diese „Multiplizierung der Akteur_innen, Orte und Praktiken“ (Heimeshoff et al. 2014, S. 15) von Grenzen begrifflich fassen zu können. Infolge Heimeshoff et al. sind Grenzen für ihr Bestehen und ihre Effektivität von ritualisierten, sich ständig wiederholenden „alltäglichen Mikropraktiken“ (ebd., S. 15; siehe auch Salter 2011) abhängig, bei denen zunehmend auch Maschinen eine wichtige Rolle spielen. Die Grenze tritt in der Analyse von Heimeshoff et al. (ebd., S. 15) als „ein performativer Akt“ hervor, an dem sowohl „menschliche und nicht-menschliche Akteur_innen beteiligt sind“. Die Autoren folgen hier einem erweiterten Souveränitätsbegriff im Sinne Butlers (2004) und Salters (2011) und erweitern ihn um die Dimension maschineller Akteure.

Grenzen und Grenzregime können demnach nur durch die Kombination einer Vielfalt theoretischer und methodischer Annäherungen sinnvoll erfasst werden. Ziel solcher fächerübergreifenden Ansätze ist es nicht, einen traditionellen politikwissenschaftlichen Fokus auf internationale Relationen und staatliche Institutionen und Akteure zu ersetzen, sondern ihn durch praxisnahe qualitative Studien sowie technologiekritische Analysen und Modelle zu ergänzen. Umfassende Studien individueller und kollektiver Grenzpraktiken und der sie bedingenden technisch-institutionellen Rahmen sind für ein Verständnis heutiger Grenzregime von entscheidender Bedeutung. Karine Côté-Boucher et al. (2014) nehmen solche Beobachtungen zum Anlass, um für eine Wende in Grenzstudien hin zu detaillierten Untersuchungen von situierten Alltagspraktiken des Aus- und Eingrenzens zu werben, während Salter (2013) für eine fachliche Wende hin zu einem practice turn argumentiert (siehe auch Brambilla 2014; Pötzsch 2018).

Eine Reihe von Studien hat eine solche Praxiswende mit Fokus auf Grenztechnologie sowie deren Funktionsweisen und Anwendungen bereits vollzogen. Walters (2011) beispielsweise zeigt, dass alltägliche Prozesse wie das Abnehmen von Fingerabdrücken oder die Anpassung von Passbildern an geltende Normen großes Potenzial für Fehler und Ungenauigkeiten beinhalten, die, in gewissen Fällen, selbst scheinbar perfekte technische Lösungen unterlaufen und aushebeln können. Bigo (2014) legt eine Studie über die diskursiven und institutionellen Rahmen vor, die die tagtäglichen Aktivitäten von Beamten, Militärs und Datenanalytikern, die mit europäischem Grenzschutz beauftragt sind, prädisponieren und daher in eine bestimmte Richtung beeinflussen (siehe auch Allen/Vollmer 2018). Die Feldstudie über Alltagspraktiken 
in britischen Auffanglagern für Flüchtlinge von Alexandra Hall (2012) wiederum zeigt auf, wie technische Lösungen die Wahrnehmungen und Praktiken von Angestellten beeinflussen, und öffnet den Blick für eine Reihe an Gegenmaßnahmen, mit deren Hilfe Migranten versuchen, sowohl Kontrollen zu unterlaufen als auch eine endgültige Registrierung hinauszuzögern oder gar ganz zu umgehen. Ihre Studie deckt überraschende Fälle von Loyalitätskonflikten und unerwarteten Hilfestellungen auch von in den Zentren angestellten Beamten auf und leuchtet deren Verständnis und Anwendung von Regelwerken und Technologie kritisch aus. Aus ähnlicher Sicht zeichnet Ruben Andersson (2014) die Migrationswege afrikanischer Auswanderer auf ihrem Weg gen Europa nach und bringt deren Praktiken mit dem Wirken einer globalen privatwirtschaftlichen Migrationsindustrie in Zusammenhang. Dadurch verbindet er die in seiner ethnografischen Feldstudie dargestellten konkreten Migrationspraktiken mit einem kritischen Verständnis der diese Praktiken bedingenden politischen Ökonomie.

Konkret situierte Praktiken haben auch Einfluss auf die Funktionstüchtigkeit und damit die Legitimität von prädiktiver Analytik als Komponente heutiger Grenzregime (vgl. Hess/ Schmidt-Sembdner in diesem Band). Tsianos und Kuster (2012) berichten in einem breit angelegten Rapport über die gemeinsame europäische Fingerabdruckdatenbank Eurodac von Grenzlokationen in Deutschland, Italien und Griechenland. Die Verfasser zeigen, wie technische Probleme, Datenschutzverordnungen, variierende nationale Standards und Leistungsvermögen sowie effektive Gegenmaßnahmen der prozessierten Individuen und Gruppen zu fehlerhaften Registrierungen führen können. Auf diese Weise können sich eingangsweise kleine Fehler schnell durch Reihen dicht verknüpfter Datenbanken verpflanzen und so auf lange Sicht zu signifikanten Fehldarstellungen und falschen Entscheidungen führen (für ähnliche Fehlerquellen am Beispiel des US-amerikanischen Drohnenkrieges siehe Scahill/Devereaux 2014 sowie Scahill/Greenwald 2014). Diese Beispiele belegen die Anfälligkeit von einzig auf kontextlose Muster in großen Datensets rekurrierender, prädiktiver algorithmischer Analytik für solche zu Beginn scheinbar unbedeutende Verzerrungen in der Dateneingabe.

An den deterritorialisierten und verkörperten Grenzen heutiger Sicherheitsregime macht eine weitgehend automatisierte Analyse von implizit als vollständig und valid angesehenen riesigen Datensets chaotische Informationsmengen handhabbar und daher operativ. Die identifizierten Muster und Abweichungen stellen so zunehmend die Grundlage staatlichen Handelns und Denkens dar und werden für die konkreten Lebenswelten der prozessierten Individuen relevant. Diese Prozesse der Operationalisierung, und ihre Anfälligkeit für Fehler und Missbrauch wiederum sind der Kern einer konstitutiven Funktion heutiger datengetriebener Grenzregime.

\subsection{Subjektivitäten, data doubles und konstitutive Effekte: spätmoderne Grenzregime als Kulturtechnik}

Wie dieser Beitrag bisher gezeigt hat, sind hochtechnologische spätmoderne Grenzregime deterritorialisiert, individualisiert, verkörpert und in ihrer Funktionstüchtigkeit von konkret situierten Alltagspraktiken der Anwendung und Instandhaltung abhängig. Neben diesen Faktoren, die staatliche Regulierungs- und Sortierungsfunktionen entgrenzen und in Alltagspraktiken und Privatsphäre verankern, treten heutige Grenzen jedoch auch als zunehmend prädiktiv und auf eine lediglich mögliche Zukunft ausgerichtet hervor. 
Eine Verkoppelung digitaler Überwachungs- und Netzwerktechnologie mit interoperativen Datenbanken ermöglicht algorithmisch getriebene Analysen großer Datenmengen, die sich als abstrakte Identitätsmuster in prädiktiven digitalen Profilen auskristallisieren. Solche Verfahren dienen der Regulierung und Kontrolle von abstrakten Verhaltens- und Assoziationsmustern, die zunehmend auf eine wahrscheinliche oder lediglich mögliche Zukunft ausgerichtet sind (vgl. Mueller 2008; Amoore 2013; Pötzsch 2015).

In diesen Prozessen spielen mit Hilfe von Mustererkennungsalgorithmen ermittelte sogenannte data doubles (vgl. Mueller 2008) eine zentrale Rolle. Diese in digitalen Datenbanken gelagerten Identitätspotenziale bilden die Basis für staatlich sanktioniertes Handeln wie das Einund Ausgrenzen von Migranten oder das Inhaftieren und Töten möglicher Terroristen. Ein digital ermitteltes, rein fragmentarisches und kontingentes Identitätspotenzial fungiert damit als Grundlage für Handlungen, die konkrete Auswirkungen auf die gelebten Realitäten und Lebenschancen der prozessierten Individuen haben. In solchen Rückkopplungen zwischen algorithmisch erarbeiteten Profilen und gelebten Identitäten liegt der Kern eines konstituierenden Effekts dieser Technologien.

Algorithmisches Sortieren und Profilieren menschlicher Daten wirkt insofern auch performativ. Technologische Praktiken nehmen an der Verwirklichung der Möglichkeiten Teil, die sie nur zu beschreiben behaupten. Rita Raley (2013, S. 128) drückt diese Zusammenhänge folgendermaßen aus: „the composition of flecks and bits of data into a profile of a terror suspect, the re-grounding of abstract data in the targeting of an actual life, will have the effect of producing that life, that body, as a terror suspect." Ein solches Verständnis der Konsequenzen digitaler Profile und algorithmischer Analytik hat Folgen für deren Legitimität als Werkzeuge in heutigen Grenzregimen.

Wie Amoore (2013) belegt, verschiebt algorithmische Prädiktion einen staatlichen Auftrag zur Sicherung von Grenzen und Gesellschaften vor konkreten Bedrohungen hin zur Sicherung einer nur noch wahrscheinlichen Zukunft, die im Prinzip als von überall her und dauernd bedroht erscheint. Die oben genannten Technologien machen nicht nur eine mögliche Zukunft zugänglich, sie tragen durch stetige Rückkoppelungen auch aktiv zu deren direkter Erschaffung bei. Solche Entwicklungen erfordern neue begriffliche Rahmen, um sie adäquat erfassen, kritisch hinterfragen und sich ihnen aktiv widersetzen zu können. Mit diesem Ziel vor Augen habe ich den Begriff iBorder eingeführt und ihn als eine fundamentale Kulturtechnik der Beund Ausgrenzung im Sinne Geoffrey Winthrop-Youngs (2013) definiert.

Der Begriff iBorder (Pötzsch 2015, insbesondere S. 110-112) beschreibt die Affordanzen komplexer soziotechnischer Netzwerke, die Individuen in vielfältiger Weise in grenzbezogene Prozesse einbinden. Diese Einbindung geschieht entlang der Achsen Biometrie, Netzwerküberwachung und automatisierte Analytik und wird durch eine Reihe spezifischer Dynamiken realisiert: iBorder 1) informationalisiert den menschlichen Körper, indem sie ihn in Serien von auf verkoppelten Datenbanken gelagerte digitale Profile fragmentiert, 2) individualisiert grenzbezogene Prozesse, indem sie den menschlichen Körper zum Träger einer im Prinzip unüberwindlichen Überallgrenze macht, 3) impliziert Individuen in diesen Prozessen, da sie auf stetig generierte Hintergrunddaten über Alltagshandlungen und -relationen rekurriert, um Abweichungen von etablierten Normen zu identifizieren und 4) wirft diese Abweichungen über interaktive Rückkoppelungen auf die analysierten Identitäten zurück und operationalisiert sie so. Dies führt zu einer allgegenwärtigen, jedoch nur bedingt sichtbaren und fühlbaren Grenze, die nicht nur bestimmte Identitäten und Verhaltensmuster identifiziert, sondern diese auch 
aktiv selbst konstituiert. Hier zeigt staatliche Macht ihre produktiven Qualitäten im Sinne Foucaults (2004, S. 240-250) und wird als ein sich selbst perpetuierender Prozess der Bedrohungserkennung und -verwirklichung verstehbar.

Solche konstituierenden Effekte einer Kombination von Netzwerküberwachung, Datenverkopplung sowie automatisierter Informationsbeschaffung und -operationalisierung treten als Kernelemente eines Verständnisses spätmoderner Grenzregime und -praktiken als grundlegende Kulturtechnik hervor (vgl. Pötzsch 2015). Winthrop-Young (2013) beschreibt Kulturtechnik entlang dreier ineinandergreifender Achsen: Zum Ersten umfasst der Begriff konkrete Agrartechnologien wie z.B. Bewässerungssystemen oder Viehzucht, zum Zweiten beinhaltet Kulturtechnik die konkreten Fertigkeiten und Kompetenzen, die für Verständnis und Nutzung technischer Hilfsmittel notwendig sind, zum Dritten beschreibt der Begriff grundlegende Verhältnisse von Mensch und Technologie. Nach Winthrop-Young (2013) bedingen sich z.B. in der Kulturtechnik des Schreibens Autor, Bleistift und Papier gegenseitig. Damit wird die Problematisierung eines überkommenen, implizit hierarchischen Agensbegriffs - das Subjekt Mensch nutzt das Objekt Bleistift - möglich. Agens entsteht durch die situierte Interaktion variierender agentic capacities - sich wechselweise bedingender und in stetiger Änderung begriffener Handlungsmöglichkeiten und -horizonte, die sowohl menschliche als auch nichtmenschliche Akteure miteinschließen. In diesem Sinne können Einheiten in soziotechnischen Netzwerken oder Assemblagen (vgl. Allen/Vollmer 2018) als Subjekte/Objekte verstanden werden, die mit anderen Subjekten/Objekten in sich gegenseitig konstituierenden Relationen stehen (vgl. Coole 2013; Pötzsch 2017). In heutigen Grenzregimen können die konstituierenden Effekte prädiktiver Analytik auf individuelle Identitäten und Handlungen als eine Kulturtechnik des In- und Ausgrenzens verstanden werden, die nicht nur faktisch Touristen und Terroristen voneinander scheidet, sondern diese Kategorien durch Rückkoppelungen mit konkreten Lebenswelten auch erst zum Erscheinen bringt.

\section{Fazit}

In einer üppig bebilderten Hochglanzbroschüre mit dem Titel Crossing Boundaries: Emerging Technologies at the Border wirbt der globale Anbieter von Hightech-Sicherheits- und Analytiklösungen Accenture für die Einführung neuester Technologien in Grenz- und Zollkontrollen. ${ }^{5}$ Auf der Titelseite prangt die Nahaufnahme eines in kaltem Blau gehaltenen menschlichen Auges, dessen Pupille die Form einer Kameralinse angenommen hat. Der Text warnt in bekannt alarmierendem Stil vor einem unmittelbar bevorstehenden Zurückfallen westlicher Grenzschutzkapazitäten hinter unaufhaltbare technologische und gesellschaftliche Entwicklungen, die sowohl neue Bedrohungen als auch effizienzsteigernde Möglichkeiten eröffnen.

In vielerlei Hinsicht sind Inhalt und Ausformung von Accentures Broschüre symptomatisch für die in diesem Beitrag behandelten Prozesse, Praktiken und Tendenzen. Vorgelegt von einem multinationalen Unternehmen mit fast 400.000 Angestellten in mehr als 120 Ländern, wirbt das Schriftstück für ein engeres Verweben biometrischer Identifikationstechnologien mit prädiktiver Analytik und automatisierten Entscheidungsprozessen an den Grenzen hochtechnisierter Industriestaaten und warnt vor den angeblich unumgänglichen negativen Konsequenzen sollten diese zugegebenerweise kostspieligen Lösungen für schlecht oder gar nicht definierte

5 Die Broschüre kann unter folgender URL eingesehen werden: www.accenture.com/us-en/insight-border-agencies-e merging-technologies, 04.06.2019. 
Herausforderungen nicht umgehend in Angriff genommen werden. Der Text spielt dabei bewusst auf ein verbreitetes Gefühl von Macht- und Einflusslosigkeit gegenüber sich schnell entwickelnden Technologien an und unterschlägt mögliche negative Implikationen und Konsequenzen der beworbenen Lösungen.

Zukünftige auf Technologie ausgerichtete Grenzforschung sollte sich solchen und ähnlichen Herausforderungen stellen. Zum einen gilt es, die oft unzureichend belegten Bedrohungsszenarien eines globalen grenzindustriellen Komplexes zu hinterfragen, um wirklichen Bedarf an neuer Technologie von rein wirtschaftlichen Interessen zu scheiden; zum anderen bedürfen neue Dynamiken wie die eines implizit konstituierenden iBordering (Pötzsch 2018) sorgfältiger empirischer Studien, um einen möglichen Einfluss auf Identitäten, Alltagspraktiken und Grenzregime empirisch zu belegen. Methodisch können dabei kritische Studien die variierenden Affordanzen gegenwärtiger und geplanter Grenzsicherungstechnologien systematisch erfassen und so mögliche gesellschaftliche und politische Implikationen herausarbeiten. Diese soziotechnischen Potenziale können dann mit qualitativen und quantitativen Datensätzen zur praktischen Implementierung, Instandhaltung sowie zu möglichen Fehlleistungen und Widerständen abgeglichen werden, um herauszufinden, ob und wie diese Potenziale in konkret gegebenen Kontexten zur Entfaltung kommen und wie diese Prozesse diskursiv gerahmt werden. Auf diese Weise kann Grenzforschung die Wirkungsweisen und Konsequenzen neuer Technologien in fächer- und methodenübergreifenden Annäherungen kritisch ausleuchten.

\section{Weiterführende Literatur}

Ajana, Btihaj (2013): Governing Through Biometrics: The Biopolitics of Identity. London: Palgrave Macmillan.

Amoore, Louise (2013): The Politics of Possibility: Risk and Security beyond Probability. Durham: Duke University Press.

Heimeshoff, Lisa-Marie/Hess, Sabine/Kron, Stefanie/Schwenken, Helen/Trzeciak, Miriam (Hrsg.) (2014): Grenzregime II: Migration, Kontrolle, Wissen. Transnationale Perspektiven. Berlin und Hamburg: Assoziation A.

Longo, Matthew (2017): The Politics of Borders: Sovereignty, Security and the Citizen After 9/11. Cambridge: Cambridge University Press.

O’Dowd, Liam (2010): From a 'Borderless World' to a 'World of Borders': Bringing History Back In: Environment \& Planning D: Society \& Space 28, H. 6, S. 1031-1050.

\section{Literaturverzeichnis}

Agamben, Giorgio (1998): Homo Sacer: Sovereign Power and Bare Life. Stanford: Stanford University Press.

Agnew, John (1994): The Territorial Trap: The Geographical Assumptions of International Relations Theory. In: Review of International Political Economy 1, H. 1, S. 53-80.

Ajana, Btihaj (2013): Governing Through Biometrics: The Biopolitics of Identity. London: Palgrave Macmillan.

Allen, William L./Vollmer, Bastian A. (2018): Clean Skins: Making the e-Border Security Assemblage. In: Environment \& Planning D: Society \& Space 36, H. 1, S. 23-39.

Amoore, Louise (2006): Biometric Borders: Governing Mobilities in the War on Terror. In: Political Geography, 25, H. 3, S. 336-351.

Amoore, Louise (2013): The Politics of Possibility: Risk and Security beyond Probability. Durham: Duke University Press.

Amoore, Louise (2018): Cloud Geographies: Computing, Data, Sovereignty. In: Progress in Human Geography 42, H. 1, S. 4-24.

Anderson, Benedict (1991): Imagined Communities: Reflections on the Origin and Spread of Nationalism. London: Verso.

Andersson, Ruben (2014): Illegality, Inc.: Clandestine Migration and the Business of Bordering Europe. Oakland: University of California Press. 
Appadurai, Arjun (1996): Modernity at Large: Cultural Dimensions of Globalization. Minneapolis: University of Minnesota Press.

Bauman, Zygmunt/Bigo, Didier/Esteves Paulo/Guild, Elspeth/Jabri Vivienne/Lyon David/Walker R.B.J. (2014): After Snowden: Rethinking the Impact of Surveillance. In International Political Sociology 8, H. 2, S. 121-144.

Bigo, Didier (2007): Detention of Foreigners, States of the Exception, and the Social Practices of Control of the Banopticon. In: Rajaram, Prem Kumar/Grundy-Warr, Carl (Hrsg.): Borderscapes: Hidden Geographies and Politics at Territory's Edge. Minneapolis: University of Minnesota Press, S. 3-33.

Bigo, Didier (2014): The (In)Securitization Practices of the Three Universes of EU Border Control: Military/Navy - Border Guards/Police - Database Analysts. In: Security Dialogue 45, H. 8, S. 209-225.

Billé, Franck (2018): Skinworlds: Borders, Haptics, Topologies. In: Environment \& Planning D: Society \& Space 36, H. 1, S. 60-77.

Brambilla, Chiara (2014): Exploring the Critical Potential of the Borderscapes Concept. In: Geopolitics 20, H. 1, S. 14-34.

Bratton, Benjamin H. (2015): The Stack: On Software and Sovereignty. Cambridge: MIT Press.

Butler, Judith (2004): Precarious Life: The Powers of Mourning and Violence. London: Verso.

Castells, Manuel (1996): The Rise of the Network Society. Cambridge: Blackwell.

Coole, Diane (2013): Agentic Capacities and Capacious Historical Materialism: Thinking with New Materialisms in the Political Sciences. In: Millennium: Journal of International Studies 41, H. 3, S. 451-469.

Côté-Boucher, Karine/Infantino, Federica/Salter, Mark B. (2014): Border Security as Practice: An Agenda for Research. In: Security Dialogue 45, H. 3, S. 195-208.

Dahinden, Janine (2009): Are we all transnationals now? Network Transnationalism and Transnational Subjectivity: The Differing Impacts of Globalization on the Inhabitants of a Small Swiss City. In: Ethnic and Racial Studies 32, H. 8, S. 1365-1386.

Deleuze, Gilles (1992): Postscript on the Societies of Control. In: October 59, S. 3-7.

Foucault, Michel (1977): Discipline and Punish: The Birth of the Prison. New York: Pantheon Books.

Foucault, Michel (2004): Society Must Be Defended: Lectures at the Collège de France 1975-76. London: Penguin Books.

Galloway, Alexander/Thacker, Eugene (2007): The Exploit: A Theory of Networks. Minneapolis: University of Minnesota Press.

Gibson, James J. (1979): The Ecological Approach to Visual Perception. Boston: Houghton Mifflin Harcourt.

Hall, Alexandra (2012): Border Watch: Cultures of Immigration, Detention and Control. London: Pluto Press.

Hardt, Michael/Negri, Antonio (2000): Empire. Cambridge: Harvard University Press.

Heintel, Martin/Musil, Robert/Stupphann, Markus/Weixlbaumer, Norbert (2018): Grenzen: Eine Einführung. In: Heintel, Martin/Musil, Robert/Weixlbaumer, Norbert (Hrsg.): Grenzen: Theoretische, konzeptionelle und praxisbezogene Fragestellungen zu Grenzen und deren Überschreitungen. Wiesbaden: Springer, S. 1-15.

Henry, Nicholas (2018): Asylum, Work, and Precarity: Bordering the Asian-Pacific. Cham: Palgrave MacMillan.

Heimeshoff, Lisa-Marie/Hess, Sabine/Kron, Stefanie/Schwenken, Helen/Trzeciak, Miriam (2014): Einleitung. In: Heimeshoff, Lisa-Marie/Hess, Sabine/Kron, Stefanie/Schwenken, Helen/Trzeciak, Miriam (Hrsg.): Grenzregime II: Migration, Kontrolle, Wissen. Transnationale Perspektiven. Berlin und Hamburg: Assoziation A, S. 9-39.

Hogan, Mél (2015): Data Flows and Water Woes: The Utah Data Center. In. Big Data \& Society 2, H. 2. DOI: $10.1177 / 2053951715592429$.

Joseph, Daniel (2018): The Time Canada Wanted Its Own Internet. Motherboard: Net Neutrality, 13. Juni. motherboard.vice.com/en_us/article/wjbbzq/canada-wanted-its-own-internet-in-the-70s, 20.08.2020.

Kuster, Brigitta/Tsianos, Vassilis S. (2013): How to Liquefy a Moving Body: Eurodac und die Digitalisierung der Europäischen Grenze. In: Arbeitsgruppe Informatik in Bildung und Gesellschaft (Hrsg.): Biometrische Identitäten und ihre Rolle in den Diskursen um Sicherheit und Grenzen. Tagung, 30.11./1.12.2013. Berlin: Humboldt Universität, S. 19-36.

Limonier, Kevin (2017): Russia's Homegrown Web. In: Le Monde Diplomatique, Oktober 2017, S. 17-18.

Longo, Matthew (2017): The Politics of Borders: Sovereignty, Security and the Citizen After 9/11. Cambridge: Cambridge University Press.

Maguire, Mark (2009): The Birth of Biometric Security. In: Anthropology Today 25, H. 2, S. 9-14

Mueller, Benjamin J. (2008): Travellers, Borders, Dangers: Locating the Political at the Biometric Border. In: Mark B. Salter (Hrsg.): Politics at the Airport. Minneapolis: University of Minnesota Press, S. 127143. 
Nissel, Heinz (2018): Grenzen als Konstante in der Politischen Geographie und Geopolitik. In: Heintel, Martin/Musil, Robert/Weixlbaumer, Norbert (Hrsg.): Grenzen: Theoretische, konzeptionelle und praxisbezogene Fragestellungen zu Grenzen und deren Überschreitungen. Wiesbaden: Springer, S. 65-88.

Norman, Donald (1988): The Design of Everyday Things. New York: Basic Books.

O'Dowd, Liam (2010): From a 'Borderless World' to a 'World of Borders': Bringing History Back In: Environment \& Planning D: Society \& Space 28, H. 6, S. 1031-1050.

Perkins, Chris/Chris Rumford (2013): The Politics of (Un)fixity and the Vernecularisation of Borders. In: Global Society 27, H. 3, S. 267-282.

Popescu, Gabriel (2011): Bordering and Ordering in the 21st Century: Understanding Borders. New York: Rowman \& Littlefield.

Pötzsch, Holger (2015): The Emergence of iBorder: Bordering Bodies, Networks, and Machines. In: Environment \& Planning D: Society \& Space 33, H. 1, S. 101-118.

Pötzsch, Holger (2017): Media Matter. TripleC: Communication, Capitalism \& Critique 15, H. 1, S. 148 170.

Pötzsch, Holger (2018): iBorder/ing. In: Lury, Celia/Uprichard, Emma (Hrsg.): Routledge Handbook of Interdisciplinary Research Methods. London: Routledge, S. 99-103.

Pugliese, Joseph (2010): Biometrics: Bodies, Technologies, Biopolitics. London: Routledge.

Raley, Rita (2013): Dataveillance and Counterveillance. In: Gitelman, Lisa (Hrsg.): Raw Data Is an Oxymoron. Cambridge: MIT Press, S. 121-145.

Rovisco, Maria (2010): Reframing Europe and the Global: Conceptualizing the Border in Cultural Encounters. In: Environment \& Planning D: Society \& Space 28, H. 6, S. 1015-1030.

Rumford, Chris (2008): Introduction: Citizens and Borderwork in Europe. In: Space \& Polity 12, H. 1, S. 1-12.

Rumford, Chris (2012): Towards a Multiperspectival Study of Borders. In: Geopolitics 17, H. 4, S. 887902.

Salter, Mark B. (2011): Places Everyone! Studying the Performativity of the Border. In: Political Geography 30, H. 2, S. 66-67.

Salter, Mark B. (2013): The Practice Turn. In: Salter, Mark B./Mutlu, C. E. (Hrsg.): Research Methods in Critical Security Studies: An Introduction. London: Routledge, S. 85-92.

Scahill, Jeremey/Devereaux, Ryan (2014): Blacklisted: The Secret Government Rulebook for Labelling You a Terrorist, 23. Juli. The Intercept. firstlook.org/theintercept/article/2014/07/23/blacklisted/, 20.08.2020

Scahill, Jeremy/Greenwald, Glenn (2014): The NSA's Secret Role in the U.S. Assassination Program, 10. Februar. The Intercept. firstlook.org/theintercept/article/2014/02/10/the-nsas-secret-role/, 20.08.2020.

Schmitt, Carl (1922/2005): Political Theology: Four Chapters on the Concept of Sovereignty. Chicago: University of Chicago Press.

Starosielski, Nicole (2015): The Undersea Network. Durham: Duke University Press.

Tsianos, Vassilis S./Kuster, Brigitta (2012): Thematic Report: 'Border Crossings' (Mig@net: Tansnational Digital Networks, Migration and Gender). cordis.europa.eu/docs/results/244/244744/143671841-8_en. zip, 25.11.2019.

Walters, William (2011): Rezoning the Global: Technological Zones, Technological Work and the (Un-)Making of Biometric Borders. In: Squire, Vicki (Hrsg.): The Contested Politics of Mobility: Borderzones and Irregularity. London: Routledge, S. 51-73.

Winthrop-Young, Geoffrey (2013): Cultural Techniques: Preliminary Remarks. In: Theory, Culture \& Society 30, H. 6, S. 3-19. 